Stephan Grohs

\title{
Lokale Wohlfahrtsarrangements zwischen Beharrung und Wandel: Die widersprüchlichen Effekte von Ökonomisierung und Kontraktmanagement
}

\begin{abstract}
Zusammenfassung
Der Beitrag beschäftigt sich am Beispiel der Jugendhilfe empirisch mit dem Einfluss der Modernisierungs- und Ökonomisierungsimpulse auf die Ausgestaltung und den Wandel lokaler Wohlfahrtsarrangements. Im Mittelpunkt steht erstens die Frage, welchen Einfluss Modernisierungsanstrengungen der kommunalen Sozialverwaltung und Ökonomisierungsimpulse auf die institutionelle Ausprägung lokaler Wohlfahrtsproduktion, d.h. die Trägerstrukturen sozialer Dienstleistungen haben. Zweitens wird untersucht, wie die Leistungsbeziehungen und Koordinationsstrukturen zwischen Sozialverwaltung und externen Wohlfahrtsproduzenten im Zuge der Modernisierung verändert werden. Angesichts offensichtlicher Widersprüche zwischen formaler Implementierung von Instrumenten des Kontraktmanagements und den tatsächlichen Wirkungen entwikkelt der Beitrag ein Modell unterschiedlicher Implementationsstrategien.
\end{abstract}

Schlagworte: Verwaltungsmodernisierung, Kontraktmanagement, Ökonomisierung, Sozialverwaltung, Jugendhilfe

\begin{abstract}
Local Welfare Provision Between Persistence and Change: Contradictiory Effects of Contractualisation and Marketization

The article explores the impact of administrative modernisation and marketization on the provision of social services in the exemplary field of youth welfare services and childcare. It deals with the effects on the institutional provision of services and the transformation of the relationship between local governments and charities as well as private providers of services. Facing apparent contradictions between the formal implementation of contract management and observed effects a model of divergent implementation strategies is developed.
\end{abstract}

Key words: Administrative Reform, Contractualisation, Marketization, Social Administration, Youth Welfare

\section{Einleitung}

Vorliegender Beitrag beschäftigt sich am Beispiel der Jugendhilfe empirisch mit dem Einfluss der Modernisierungs- und Ökonomisierungsimpulse auf die Ausgestaltung und den Wandel lokaler Wohlfahrtsarrangements. Unter Wohlfahrtsarrangements werden hier die von Franz-Xaver Kaufmann als „Arrangements der Wohlfahrtsproduktion“ bezeichneten „Konfiguration(en) zwischen staatlichen, marktlichen, verbandlichen und privaten Formen der Wohlfahrtsproduktion" (Kaufmann 2003, S. 42) verstanden.

Das Neue Steuerungsmodell (NSM) und insbesondere das Instrument des Kontraktmanagements sollten die Leistungstransparenz erhöhen und die freien Träger einerseits an kommunale Ziele binden, andererseits aber durch Budgetierung und marktähnliche Preis- 
bildung die Träger zu effizienterem und autonomerem Management anhalten. Neben der Ausstrahlung der Binnenreformen ging erheblicher Druck von bundespolitischen Interventionen mit dem Ziel der Aufhebung des bedingten Vorrangs freier Träger sowie der Einführung neuer Finanzierungsformen aus. Zielsetzung war hier eine Pluralisierung des Anbieterspektrums und eine Intensivierung des Wettbewerbs zwischen ihnen (,Fachlich regulierter Qualitätswettbewerb" (BMSFJ 2002)), wovon man sich in erster Linie die Aktivierung von Effizienzreserven erhoffte. Kontraktmanagement und die Möglichkeit neuer Finanzierungsformen über Leistungsvereinbarungen stellten gewissermaßen eine Übertragung der binnenorientierten Modernisierungslogik auf die Steuerung freier Träger dar.

Im Mittelpunkt des Beitrags steht erstens die Frage, welchen Einfluss diese Modernisierungsanstrengungen der kommunalen Sozialverwaltung und Ökonomisierungsimpulse auf die institutionelle Ausprägung lokaler Wohlfahrtsproduktion, d.h. die Trägerstrukturen sozialer Dienstleistungen haben. Zweitens wird untersucht, wie die Leistungsbeziehungen und Koordinationsstrukturen zwischen Sozialverwaltung und externen Wohlfahrtsproduzenten im Zuge der Modernisierung verändert werden. Konkreter: Kommt es tatsächlich zu Änderungen hinsichtlich Steuerung und Kontrolle sozialer Dienste?

Nach einer Einführung in die Problemstellung und den bisherigen Stand der Diskussion (2.) und einer Skizze des methodischen Vorgehens (3.) werde ich in einem ersten empirischen Schritt anhand quantitativer Daten die Annahmen zum Wandel von Wohlfahrtsarrangements überprüfen (4.). Entgegen einiger Annahmen aus der Literatur zeigen diese Ergebnisse eine weitgehende Kontinuität in den Trägerstrukturen trotz (oder gerade wegen) Verwaltungsmodernisierung und der Implementierung kontraktbasierter Steuerungsformen auf. Daher werde ich im zweiten Schritt basierend auf vier Fallstudien versuchen, diesen zunächst überraschenden Fakt zu erklären. Hier stellt sich insbesondere die Frage nach den tatsächlichen Implementationsweisen für die Einführung von Kontrakten. Entgegen der vom Gesetzgeber beabsichtigten Wirkung der Ermöglichung von mehr Wettbewerb und Pluralität unter den Anbietern werden, so meine zentrale These, Kontrakte und fachpolitische ,professionelle" Modernisierungsansätze vielfach genutzt, um - in durchaus subversiver Manier - auf einer neuen Basis bestehende Kooperationsbeziehungen zu stabilisieren und zu schließen (5.).

\section{Konzeptioneller Rahmen und Forschungsstand}

\subsection{Konzeptioneller Rahmen}

In einer am akteurorientierten Institutionalismus angelehnten Heuristik kann das zentrale Problem des Wandels von Akteursbeziehungen in lokalen Wohlfahrtsarrangements als Institutionenwandel begriffen werden. Im Fokus stehen die dem institutionellen Set der lokalen Wohlfahrtsproduktion zugrunde liegenden Regelsysteme, für die Elinor Ostroms Unterscheidung von formalen Regeln, den ,rules in form" und informellen Regeln, den „rules in use" (vgl. Ostrom 1985, 1999) zu Grunde gelegt wurde. Die formalen Regeln „rules-in-form“ - stammen einerseits aus der Gesetzgebung, andererseits aus den Spielregeln, die sich die Kommunen selbst auferlegen (Satzungen etc.). Die „rules in use“ dagegen umfassen daneben etablierte Interaktions- und Vertrauensbeziehungen, informelle Praktiken, Normen und Tauschgeschäfte. 
Mit dieser Unterscheidung lassen sich für die Stabilität und den Wandel etablierter Strukturen eine Reihe möglicher Bestimmungsfaktoren benennen. Die oben formulierte Beharrungsthese stützt sich auf die Annahme, dass sich trotz eines Wandels der formalen Regeln die „rules in use" nicht zwangsläufig „mitwandeln". Dafür lassen sich eine Reihe möglicher Gründe benennen, die einerseits funktional zu begründen sind (Increasing Returns, Transaktionskostenminimierung, objektive Funktionalität). ${ }^{1}$ Für die entscheidenden Akteure gibt es aus dieser Perspektive schlicht keinen objektiven Grund, ihre informellen Verhaltensweisen zu ändern. Zum zweiten treten machtpolitische Faktoren hinzu: die etablierten Akteure suchen ihre jeweiligen Ressourcen und Positionen zu maximieren oder zumindest zu erhalten. Sie versuchen daher die formalen Regeln durch neue SchlieBungsmechanismen zu ,umspielen“. Eine dritte Perspektive bietet schließlich eine an der jeweiligen „logic of appropriateness" (March/Olsen 1989) orientierte Verhaltensweise, die die beschränkt rationalen Akteure weiter in ihren alten Routinen und akzeptierten Normen verharren lässt (trotz sich ändernder Umwelten).

Das alte als „neokorporatistisch“ (vgl. Heinze 1981; Heinze/Olk 1981) beschriebene Regime beruhte einerseits auf formalen Regeln (Subsidiaritätsprinzip; Vorrang der freien Wohlfahrtspflege vor nichtorganisierten Trägern; funktionale Repräsentation im Jugendhilfeausschuss; Zuwendungsfinanzierung als Selbstkostendeckung), andererseits aber auch auf informellen Regeln und Interaktionen. Hierzu zählen Tauschgeschäfte zu beiderseitigem Vorteil, Vertrauensnetzwerke, eingeschliffene Gewohnheiten etc., die die Unsicherheit zwischen den Akteuren reduzieren. Jene Unsicherheitsreduktion wird, so meine Annahme, gerade in Bereichen wichtig, wo unsichere Wirkungsketten bestehen, wie in vielen Feldern der sozialen Arbeit. Tabelle 1 fasst dieses „,alte“ Regelungswerk zusammen.

Hinsichtlich der formalen Regeln kam es seit den 1990er Jahren zu einem deutlichen Wandel (vgl. z.B. Dahme u.a. 2005, S. 38-42). Alle diese Maßnahmen beabsichtigten eine Pluralisierung der Trägerstrukturen und eine zunehmende Orientierung am Wettbewerb. ${ }^{2} \mathrm{Zu}$ nennen ist hier von gesetzgeberischer Seite die Aufhebung des bedingten Vorrangs der Freien Wohlfahrtspflege und die damit einhergehende weitgehende Gleichstellung mit privaten Anbietern in BSHG und SGB VIII, die Einführung von Leistungsvereinbarungen (statt Zuwendungsfinanzierung) im BSHG und Teilen des SGB VIII. Innerhalb der Kommunen kam es zu einer Herausforderung durch Einführung von Elementen des Neuen Steuerungsmodells, das in der Beziehung zu den Trägern sozialer Arbeit insbesondere durch die Einführung eines Kontrakt- und Qualitätsmanagement zur Änderung der formalen Regeln führt. Die neuen Anforderungen durch die Modernisierungsansätze wurden in der Regel an die fachlich segmentierten Netzwerke ,von außen" durch die „zentralen Steuerungspolitiker" (Banner 1984) herangetragen. Der Modernisierungsdruck durch die Verwaltungsspitze und Erwartungshaltungen von außen führten in einer Großzahl von Kommunen dazu, dass die Fachebene gezwungen war, sich zumindest formal mit den Modernisierungskonzepten auseinanderzusetzen. Die empirisch zu beantwortende Frage ist, ob und unter welchen Bedingungen diese Modernisierungsimpulse in den Kommunen zu Auswirkungen auf die tatsächliche Ausgestaltung der Trägerbeziehungen führen. 
Tabelle 1: Das institutionelle Set lokaler Wohlfahrtsarrangements

\begin{tabular}{|c|c|c|c|}
\hline $\begin{array}{l}\text { Regel- } \\
\text { strukturen }\end{array}$ & $\begin{array}{l}\text { Formelle Regeln des } \\
\text { alten „neokorporatistischen“ } \\
\text { Regimes }\end{array}$ & Informelle Regeln & $\begin{array}{l}\text { Formelle Regeln des neuen } \\
\text { "wohlfahrtspluralistischen } \\
\text { Regimes" seit ca. } 1999\end{array}$ \\
\hline $\begin{array}{l}\text { Positions- und } \\
\text { Zugangsregeln }\end{array}$ & $\begin{array}{l}\text { Funktionale Repräsentation } \\
\text { der Verbände im Jugendhil- } \\
\text { feausschuss (§ } 71 \text { SGB VIII) }\end{array}$ & $\begin{array}{l}\text { - Starke personelle Verflechtung } \\
\text { der Verbands-vertreter mit So- } \\
\text { zialadministration. } \\
\text { - Parteipolitische Verflechtung } \\
\text { der Verbände (AWO-SPD; } \\
\text { DCV-CDU; DPWV-Grüne) } \\
\text { - Ausschluss der Interessen von } \\
\text { Leistungsempfängern }\end{array}$ & $\begin{array}{l}\text { - Keine Änderungen (aber ab } \\
2009 \text { Auflösung der JHA- } \\
\text { Strukturen durch Landesge- } \\
\text { setzgeber zu erwarten) } \\
\text { - Potentielle Entflechtung zwi- } \\
\text { schen Administration und } \\
\text { JHA durch NSM } \\
\text { - Stärkung der Beteiligungs- } \\
\text { rechte von Leistungsemp- } \\
\text { fängern (z.B. \$5 SGB VIII) }\end{array}$ \\
\hline $\begin{array}{l}\text { Auszahlungs- } \\
\text { regeln }\end{array}$ & $\begin{array}{l}\text { - Bedingter Vorrang der Ver- } \\
\text { bände vor öffentlichen und } \\
\text { nichtorganisierten Trägern } \\
\text { (\$10 BSHG) } \\
\text { - Zuwendungsfinanzierung } \\
\text { nach Selbstkostendek- } \\
\text { kungsprinzip }\end{array}$ & $\begin{array}{l}\text { - De facto „Markt"aufteilung nach } \\
\text { Verbändeparität }\end{array}$ & $\begin{array}{l}- \text { Aufhebung des bedingten } \\
\text { Vorrangs und Gleichstellung } \\
\text { nichtorganisierter Träger } \\
\text { (z.B. §3; § 98a-g SGB VIII) } \\
\text { - Einführung von Leistungs- } \\
\text { verträgen bzW. -vereinba- } \\
\text { rungen (\$93 BSHG; §98a-g } \\
\text { SGB VIII) } \\
\text { - Prospektive Leistungsent- } \\
\text { gelte statt Selbstkostende- } \\
\text { ckungsprinzip } \\
\text { - Kontraktmanagement; z.T. } \\
\text { sozialraumorientierte Bud- } \\
\text { getierung } \\
\end{array}$ \\
\hline $\begin{array}{l}\text { Informations- } \\
\text { regeln }\end{array}$ & $\begin{array}{l}\text { Verzicht auf standardisierte } \\
\text { Qualitätskontrolle: Verwen- } \\
\text { dungsnachweise }\end{array}$ & $\begin{array}{l}\text { - (Qualitäts-) „Management by } \\
\text { Rumour" }\end{array}$ & $\begin{array}{l}\text { Output-Kontrolle durch Qua- } \\
\text { litätsmanagement und Qua- } \\
\text { litätsentwicklungsvereinba- } \\
\text { rungen ((\$93 BSHG; } \\
\text { §98a-g ff. SGB VIII) }\end{array}$ \\
\hline
\end{tabular}

\subsection{Kontraktmanagement und Wohlfahrtsproduktion}

Über die Auswirkungen der binnen- wie der außenbezogenen Modernisierungsbestrebungen auf lokale Wohlfahrtsarrangements liegen außer Praktikerberichten und Arbeiten eher normativer Provenienz nur wenige systematische Erkenntnisse vor. Vielmehr lag der bisherige Fokus der empirischen Forschung erstens auf der Umsetzung binnenorganisatorischer Reformen in den kommunalen Sozialadministrationen (vgl. Bußmann/Esch/StöbeBlossey 2003; Mamier u.a. 2002; Seckinger u.a. 1998; Pluto 2005). Zweitens wurden die Auswirkungen von Verwaltungsmodernisierung und Ökonomisierungstendenzen auf die leistungserbringenden Träger (vgl. Dahme u.a. 2005; Messmer 2007) oder die Auswirkungen auf das individuelle Handeln der Professionellen in der Sozialen Arbeit untersucht (vgl. Dahme u.a. 2005). Die Mesoebene der lokalen Vermittlungsstrukturen und das "Gesamtsystem" der lokalen Trägerlandschaft, das Kernstück lokaler Sozialpolitik, blieben demgegenüber weitgehend vernachlässigt.

Die spärliche und oft an Einzelbeispielen orientierte wissenschaftliche Literatur zu den Mesostrukturen formuliert im Kern zwei konkurrierende Thesen (vgl. Evers/Olk 1996): Auf der einen Seite wird der Wandel betont. Im Speziellen ist von einer Pluralisierung, Diversifizierung oder Ökonomisierung lokaler Wohlfahrtsarrangements und einem Niedergang traditionell-korporatistischer Verflechtungsstrukturen die Rede. Als Beispiele 
für diese Argumentationsrichtung können Thesen einer „Pluralisierung des Anbieterspektrums" (Backhaus-Maul/Olk 1994), einer „Pluralisierung und Kontraktualisierung" sozialer Dienste (Wollmann 2004, S. 17; vgl. auch Bönker/Wollmann 2006), einer „schleichenden Privatisierung“ (Evers/Rauch/Stitz 2002, S. 11) oder schlicht einer "Ökonomisierung“ (Boeßenecker u.a. 2000; 2001) gelten. Hinsichtlich der Beziehungen zu den etablierten Verbänden wird eine Ablösung des Korporatismus durch den Markt (vgl. Münder 1998, S. 11) und der Entwicklung neuer Muster eines „Postkorporatismus“ (Pabst 1998) diskutiert.

Diesen Wandlungsthesen in ihren verschiedenen Spielarten wird eine Beharrungsthese entgegengesetzt: So konstatieren Heinze und Strünck: „On a local level, however, the status quo remains and new projects are even forced to join the traditional umbrella associations" (Heinze/Strünck 2000). Allerdings kontrastieren auch sie einen „Übergang vom ,Status' zum ,Kontrakt”“ (Heinze/Schmid/Strünck 1997), der über „,betriebswirtschaftliche Reorganisation und neue Vertragsbeziehungen (...) auf Systemebene zu einer Stabilisierung und Zentralisierung führt, die verbandliche Steuerungsprinzipien stärkt" (Heinze/ Schmid/Strünck 1997, S. 249). Diese Kontinuität wird in der Regel mit den Beharrungskräften der in „neokorporatistischen" (Heinze 1981) Arrangements und „verhandlungsdemokratischen" kommunalpolitischen Strukturen (Bogumil/Holtkamp 2006) eingebetteten Verflechtung der Wohlfahrtsverbände mit den lokalen Verwaltungen und der lokalen Politik begründet. Nach dieser These werden zwar die Spielregeln geändert, die Spieler bleiben jedoch dieselben. Gerade neuere Beiträge betonen diese beharrende Tendenz gegenüber den oben zitierten (frühen) Wandlungsdiagnosen (vgl. Meyer 2008; Möhring-Hesse 2008).

Die Umstellung der Leistungsbeziehungen von einem marktfernen Subventionsmodell auf kontraktbasierte „Quasi-Märkte“ ist kein rein deutsches Phänomen. International ist diese Entwicklung im Zuge der New Public Management-Bewegung in unterschiedlichen Ausprägungen zu beobachten. Erfahrungsberichte liegen insbesondere aus dem angelsächsischen Bereich, im kontinentaleuropäischen Kontext insbesondere aus der Schweiz vor. In beiden Fällen kann von einer weitergehenden „Kontraktkultur" als im deutschen Fall gesprochen werden. Die Erfahrungen aus der angelsächsischen und Schweizer Literatur zeigen in verschiedenen Bereichen der Kontraktualisierung auf, dass auch in diesen weniger wohlfahrtskorporatistisch verfassten Systemen die Implementation der Leistungsverträge in der Regel nicht den NPM-Blaupausen entspricht.

Die Vergabe der Leistungsvereinbarungen scheint auch in der Schweiz und angelsächsischen Ländern eher auf etablierten Kooperationsstrukturen denn auf Ausschreibungswettbewerben zu basieren. Wettbewerb (z.B. durch Ausschreibungen) spielte dabei keine dominante Rolle. Für die Schweiz wird konstatiert: „Bestehende Beziehungen, Vertrauen und Stabilität bestimmen das Zustandekommen von Leistungsverträgen, nicht Marktelemente wie Preis, Quantität und Qualität" (Ruflin 2006, S. 243). Auch in den angelsächsischen Staaten bleibt trotz Privatisierung und der Übertragung sozialer Dienste der Wettbewerb häufig aus (vgl. Beinecke u.a. 1997; Johnson u.a.. 1998; Van Slyke 2003). Van Slyke kommt in einer Sekundäranalyse von 12 Studien zum Kontraktmanagement mit sozialen Diensten aus den USA zum Schluss: „There are several consistent findings across the empirical studies [...]. These include a lack of competition, administrative capacity on the part of both actors - public and nonprofit and performance measures"(Van Slyke 2003, S. 298). Angesichts dieser Entwicklung wird konstatiert, dass es kaum zu größerer Wahlfreiheit für die Klienten kam und für die Anbieter dadurch auch wenige Anreize für eine verstärkte „Kundenorientierung“ bestünden (vgl. Powell 1999). 
Als Erklärungsfaktoren für diese symptomatischen Diagnosen lassen sich aus der internationalen Literatur drei wesentliche Ursachenbündel herausarbeiten:

- Angebotsseitig eine begrenzte Zahl von Anbietern auf den lokalen Märkten (vgl. Smith/Smyth 1996, S. 282ff.; Johnson u.a. 1998, S. 318).

- Nachfrageseitig eine Reduzierung der Informations- und Suchkosten durch Kontrakte mit schon bekannten Anbietern (vgl. Johnson u.a. 1996, S. 317).

- sowie eine allgemeine Zufriedenheit mit der bisherigen Leistungserbringung und die Bevorzugung von langfristiger Programmstabilität vor kurzfristigem Wettbewerb (vgl. Johnson u.a. 1998, S. 317f;; Romzek/Johnston 2002, S. 447ff.; Smith/Smyth 1996, S. 290; Milward/Provan 2000, S. 369f.)).

Die in diesem Abschnitt aufgeworfenen Fragen zu Persistenz und Wandel von Wohlfahrtsarrangements sollen im Folgenden einer empirischen Prüfung unterzogen werden.

\section{Methodisches Vorgehen}

Auf Grund der besonderen Bedeutung in finanzieller, personeller, aber auch in strategisch-kommunalpolitischer Hinsicht sowie der besseren Vergleichbarkeit wird die Kinder- und Jugendhilfe als Untersuchungsfeld sozialer Dienste ausgewählt. Den Untersuchungszeitraum bildet die Periode von Anfang der 1990er Jahre bis 2008. Als Ausgangspunkt wurde der Beginn der Modernisierungswelle des Neuen Steuerungsmodells ab 1991 gewählt; die Ökonomisierungs- und Wettbewerbsimpulse setzten einige Jahre später ein und hatten ihren Höhepunkt Ende der 1990er Jahre. Somit liegen hinreichende Erfahrungen mit den neuen Kontraktformen vor, um deren tatsächliche Effekte empirisch zu evaluieren.

In einem ersten empirischen Schritt (vgl. Abschnitt 4) werden mit Hilfe quantitativer Daten der Wandel der Trägerstrukturen und der Einfluss von Kontraktmanagement und Leistungsvereinbarungen auf diesen Wandel quantitativ untersucht. Datenquellen dieser Untersuchung sind Daten der amtlichen Kinder- und Jugendhilfestatistik ${ }^{3}$ und Daten aus dem Forschungsprojekt „10 Jahre Neues Steuerungsmodell" ${ }^{\star 4}$, die Einblick in den Modernisierungsstand und die Ausgestaltung der Außenbeziehungen deutscher Jugendämter erlauben.

Während im ersten quantitativen Schritt die Verallgemeinerbarkeit der Ergebnisse zentral war, geht es in einem zweiten qualitativen Schritt um die Identifikation und Rekonstruktion typischer Situationslogiken und institutionenpolitischer Prozesse (vgl. Abschnitt 5). Die Fallauswahl orientiert sich daher an der Frage, wie sich Modernisierungsund Ökonomisierungstendenzen auf die lokalen Akteursbeziehungen auswirken und wie die Akteure mit den neuen Instrumenten umgehen. Zur Fallauswahl werden dabei hervorstechende Merkmalskonstellationen aus der quantitativen Untersuchung herausgegriffen und so über ein ,theoretisches Sampling“ Untersuchungskommunen bestimmt. Datengrundlage dieses Samplings ist wiederum der Datensatz aus dem Projekt „10 Jahre NSM". Alle Fälle haben gemeinsam, dass Erfahrungen mit der Umstellung auf kontraktförmige Beziehungsformen vorliegen, um Varianzen in der Umsetzung und den Auswirkungen dieser Maßnahmen enger ins Blickfeld nehmen zu können. Um die These des Einflusses neokorporatistischer Strukturen auf die Kontinuität der Trägerstrukturen zu untersuchen, werden dabei zwei west- mit zwei ostdeutschen Kommunen verglichen, da 
plausibel davon auszugehen ist, dass die Trägerstrukturen durch den Neuaufbau nach der Wende (vgl. Angerhausen u.a. 1998) in den neuen Bundesländern weniger etablierte und verfestigte Gefüge aufweisen. Innerhalb der ost- und westdeutschen Fälle wird hernach unterschieden, ob ein Wandel der Trägerstrukturen vorliegt oder nicht. Diese „kreuzweise" Fallkontrastierung soll der Identifizierung der entscheidenden Prozessvariablen dienen. Die Fälle sind dabei nicht repräsentativ für einen gleichen Anteil von Kommunen. Die Größe der Fallkommunen wurde weitgehend konstant gehalten (Städte mit eigenständigem Jugendamt zwischen 50.000 und 100.000 Einwohner). Die Fallstudien stützen sich methodisch auf Dokumentenanalysen und Experteninterviews. Dazu wurden in den Fallkommunen zwischen 2006 und 2008 insgesamt 26 Gespräche mit den Sozialdezernenten, Jugendamtsleitern, ausgewählten Mitgliedern des Jugendhilfeausschusses sowie den Geschäftsführern der jeweils dominierenden Wohlfahrtsverbände sowie gegebenenfalls eines freien Anbieters aus dem privatwirtschaftlichen bzw. bürgerschaftlichen Sektor durchgeführt.

\section{Wandel lokaler Wohlfahrtsarrangements: Halbierte Kontraktualisierung}

In einem ersten empirischen Schritt wird der Wandel der lokalen Trägerstrukturen bundesweit untersucht. Dabei werden die Trägerstrukturen sowohl in ihrer Ausprägung hinsichtlich der Anteile der verschiedenen Trägergruppen als auch hinsichtlich der institutionellen Ausgestaltung (Einführung von Kontraktmanagement, Leistungsvereinbarungen und Wettbewerbselementen) untersucht. Es stehen drei wesentliche Fragen im Mittelpunkt: Zunächst wird der tatsächliche Wandel der Trägerstrukturen untersucht, danach wird die Implementation der "neuen Regeln" nachgezeichnet und schließlich die Frage nach dem Zusammenhang neuer Regelungsstrukturen und dem Wandel bzw. der Konstanz der Trägerstrukturen gestellt.

\subsection{Wandel der Trägerstrukturen}

Betrachtet man zunächst den Wandel der Trägerstrukturen anhand der Trägerschaft der Einrichtungen, so fällt für Westdeutschland eine überraschende Kontinuität der Trägerstrukturen im Zeitverlauf auf (vgl. Abb. 1 und 2). Sowohl im Bereich der Kindertagesbetreuung wie im Bereich der Jugendhilfe sind in den alten Bundesländern über den Beobachtungszeitraum kaum Veränderungen in den Anteilen der einzelnen Trägergruppen festzustellen. Trotz Verschiebungen im Aufgabenportfolio (Ausbau Kleinstkinderbetreuung, Umstellung von stationärer zu ambulanter Unterbringung etc.) ist es den bisherigen Trägerensembles offensichtlich gelungen, ihre jeweiligen Anteile zu bewahren. Eine Ausnahme stellt das relativ neue Feld der ,Kleinsteinrichtungen der stationären Erziehungshilfe" dar, ein hoch spezialisiertes Segment, das der einzige Bereich ist, in dem privatwirtschaftliche Anbieter einen nennenswerten Anteil stellen (rund 30\% im Jahr 2002). 
Abbildung 1: Wandel der Trägerstrukturen Kindertagesbetreuung

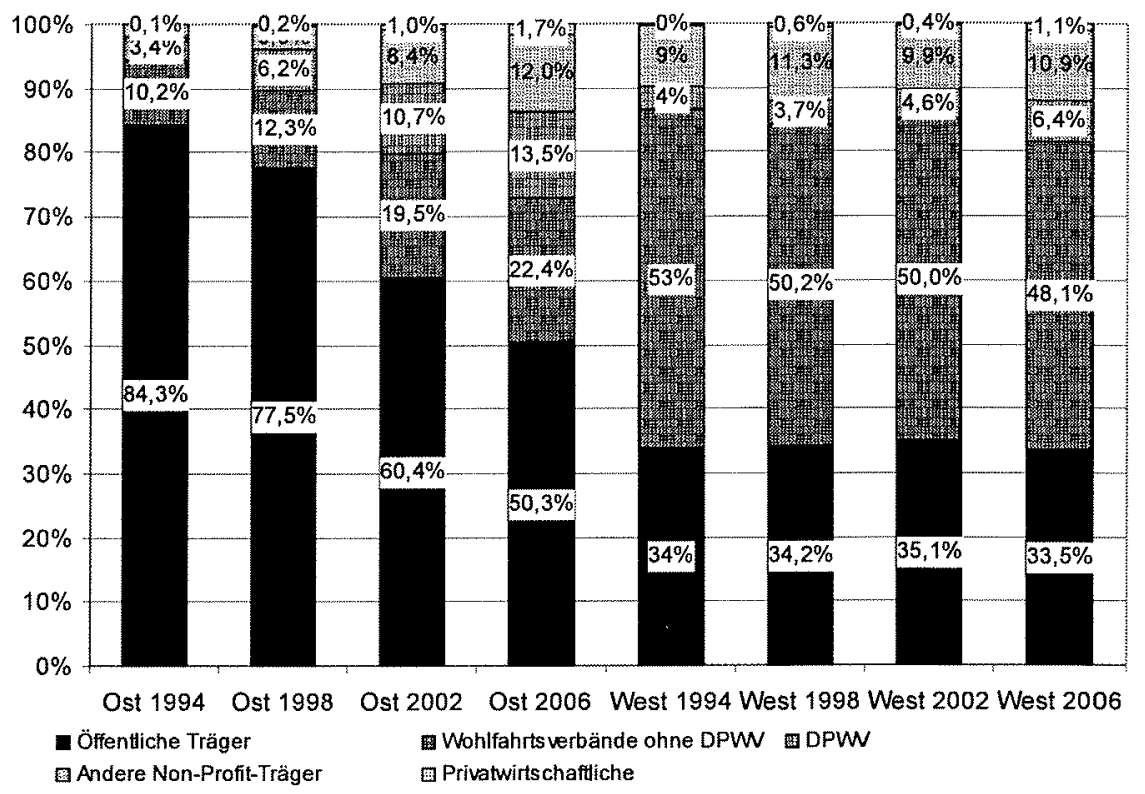

Quelle: Eigene Berechnungen auf Grundlage: Statistisches Bundesamt Fachserie 13, Reihe 6.3, Einrichtungen und tätige Personen in der Jugendhilfe. Jg. 1994, 1998, 2002, 2006. Jeweils Bonn 1994, 1998, 2002; 2007.

Abbildung 2: Wandel der Trägerstrukturen Jugendhilfe ohne Kindertagesbetreuung

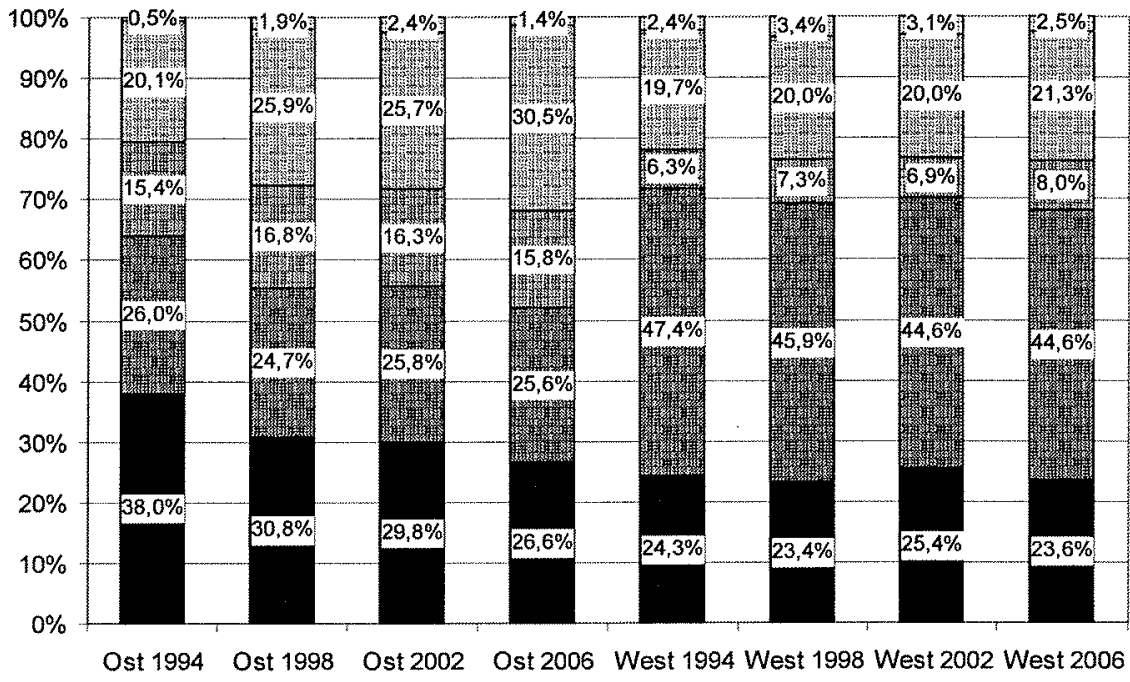

- Öffentliche Träger w Wohlfahrtsverbände ohne DPWV 圈DPW 团Andere Non-Profit-Träger 6 Privatwirtschaftiche

Eigene Berechnungen auf Grundlage: Statistisches Bundesamt Fachserie 13, Reihe 6.3, Einrichtungen und tätige Personen in der Jugendhilfe. Jg. 1994, 1998, 2002. Jeweils Bonn 1994, 1998, 2002, 2007.

Für die neuen Bundesländer ist im Zeitverlauf in erster Linie eine „Entkommunalisierung“ kennzeichnend. Von der aus DDR-Zeiten übernommenen kommunalen Eigenproduktion entwickeln sich die ostdeutschen Wohlfahrtsarrangements hin zu einer durchaus mit dem Westen vergleichbaren Gewährleistungsverwaltung. Der vom Deutschen Jugendinstitut (DJI) konstatierte Wandel weg von öffentlicher Trägerschaft (vgl. Pluto u.a. 2007, S. 14) ist 
daher in erster Linie ein ostdeutsches Phänomen, das in der Tendenz eine Annäherung an westdeutsche Verhältnisse erkennen lässt. In den alten Bundesländern bleiben zumindest nach der öffentlichen Statistik die öffentlichen Anteile weitgehend konstant. Auch die vom DJI konstatierte Zunahme privatgewerblicher Anbieter (Pluto u.a. 2007, S. 14-15) lässt sich aus der öffentlichen Statistik nur bedingt ablesen. Generell konnten sich die westdeutschen Wohlfahrtsverbände auch ein beachtliches Terrain sichern (gegeben der Tatsache, dass sie nach 1990 ihre gesamte Infrastruktur neu aufbauen mussten (vgl. Angerhausen u.a. 1998)). Der DPWV hat in Ostdeutschland durchweg größere Anteile, was zum einen durch das Aufgehen der zu DDR-Zeiten quasistaatlichen „Volkssolidarität“ im DPWV bedingt, andererseits auf das Eintreten zahlreicherer kleinerer Projekte in den DPWV zurückzuführen ist. Insgesamt zeichnen sich die ostdeutschen Strukturen dennoch durch eine größere Pluralität, allerdings einen noch geringeren Anteil privatgewerblicher Anbieter aus.

Trotz einzelner Nischen kann also hinsichtlich der "Wandlungshypothese“ zusammenfassend konstatiert werden, dass zumindest für Westdeutschland gemessen an der Trägerschaft von einer Auflösung des „Wohlfahrtskorporatismus“ nicht die Rede sein kann. Über die letzten 15 Jahre ist hier von weitgehender Kontinuität auszugehen. Für den Osten ist eine Angleichung der Strukturen an Westdeutschland zu beobachten, allerdings wird diese Tendenz durch einen höheren Anteil ,bürgerschaftlich" getragener Einrichtungen außerhalb der Wohlfahrtsverbände ergänzt. Wechselt man die Beobachtungsperspektive von der amtlichen Kinder- und Jugendhilfestatistik zu den Mikrodaten der Jugendamtsleitungen aus dem „10 Jahre NSM“-Projekt und betrachtet die Aussagen der Jugendamtsleitungen zur Übertragung von Aufgaben an Dritte in den vergangenen fünf Jahren verfestigt sich dieses Bild (vgl. Abbildung 3).

Abbildung 3: Vermehrte Übertragung von Aufgaben auf Dritte in deutschen Jugendämtern

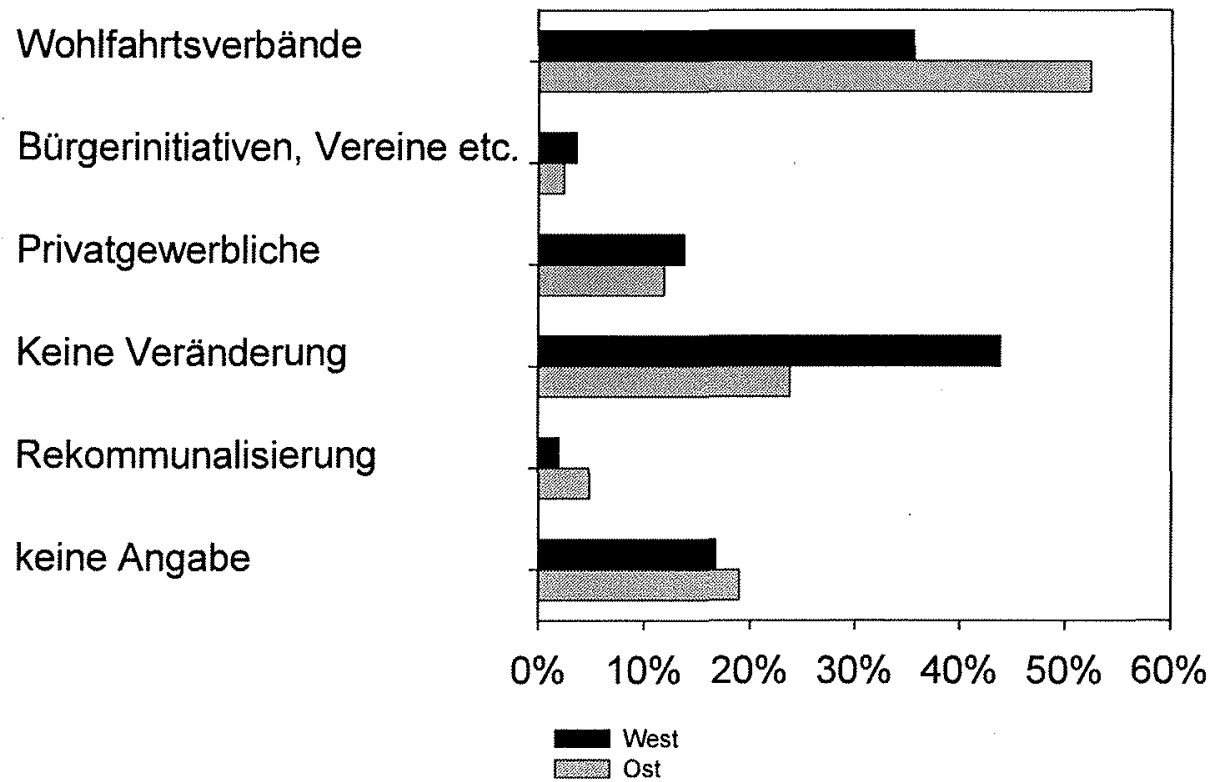

Quelle: HBS-Projekt „10 Jahre NSM“, Jugendamtsbefragung; Frageformulierung; „Wurden in den letzten 5 Jahren vermehrt Aufgaben der Kinder- und Jugendhilfe auf freie Träger und privatgewerbliche Anbieter übertragen? Wenn ja, auf welche?"; $n=241$. 
Hier zeigt sich insbesondere in Ostdeutschland ein „Aufholen“ (Angerhausen u.a. 1998) der Verbände durch eine vermehrte Übertragung auf Wohlfahrtsverbände, das Potential des nicht-verbandlichen Dritten Sektors scheint hier mittlerweile weitgehend erschöpft zu sein. Im Westen dominiert offensichtlich der Status quo, gefolgt von einem weiteren Ausbau der Verbändewohlfahrt. Privatgewerbliche Anbieter spielen hier nur im Bereich der hoch spezialisierten Hilfen zur Erziehung eine Rolle, deren Ausdehnung sich in dieser Grafik widerspiegelt. Bei letzteren handelt es sich um kleinste private Träger mit 2 bis 6 Plätzen. Häufig handelt es sich nicht um primär gewinnorientierte Unternehmen, sondern Abspaltungen größerer Träger und durch Einzelpersonen getragene Einrichtungen. Operationalisiert man Pluralisierung und Ökonomisierung mit einer verstärkten Heranziehung privatgewerblicher Anbieter einerseits, bürgerschaftlicher Initiativen und Vereine andererseits, kann man in höchstens 14 Prozent der Kommunen von einer solchen Pluralisierung ausgehen. Bürgerinitiativen und Vereine spielen dabei eine minimale Rolle, so dass im Bereich der Jugendhilfe nicht von einer „Aktivierungsstrategie“ geredet werden kann.

\subsection{Implementation von Kontrakten, Leistungsvereinbarungen und Wettbewerbselementen}

Die im vorigen Abschnitt geschilderte Kontinuität der Trägerstrukturen spricht auf den ersten Blick für die Beharrungsthese. In einem zweiten Schritt stellt sich nun die Frage ob diese Beharrung primär auf eine Nicht-Implementation der neuen Regelungsstrukturen oder vielmehr auf das Verfehlen der Pluralisierungsimpulse trotz Implementation zurückzuführen ist.

Betrachtet man zunächst die Einführung des Neuen Steuerungsmodells (NSM), dem in der Literatur (vgl. Dahme u.a. 2005; Boeßenecker u.a. 2001; Kulbach/Wohlfahrt 1996 m.w.N.) große Bedeutung für eine Pluralisierung und Ökonomisierung der Trägerlandschaft zugesprochen wird, so zeigt sich nur ein beschränkter Umsetzungserfolg. Wie an anderer Stelle ausführlicher gezeigt wurde (vgl. Bogumil u.a. 2007; Grohs 2007) hat sich das NSM nur sehr eingeschränkt in deutschen Kommunen und Jugendämtern durchsetzen können. Die Verwirklichung der NSM-Kernelemente scheint vielfach zu stocken, sich auf „Modernisierungsinseln" und den Einsatz von Einzelinstrumenten zu beschränken. Im Zusammenhang mit der Pluralisierung der Anbieterstrukturen wurde schon früh gezeigt, dass Kontraktmanagement mit freien Trägern selten tatsächlich umgesetzt wurde und dass Träger in der Entwicklung von Instrumenten nur eine unbedeutende Rolle spielten (vgl. Santen 1998).

Nach der vorliegenden Umfrage der Jugendamtsleitungen spielt Kontraktmanagement mit freien Trägern immerhin in einem runden Drittel der Kommunen eine Rolle. Die Effekte des Kontraktmanagements deuten jedoch nicht auf eine Pluralisierung, sondern im Gegenteil auf eine Stärkung der verbandlichen Wohlfahrtspflege hin (vgl. Abbildung 4). Keinerlei Zusammenhang zeigt sich hier mit der Übertragung von Aufgaben an privatgewerbliche Anbieter. Hier zeigt sich also ein erklärungsbedürftiger Befund, der in Richtung kontraintentionaler Effekte der neuen Instrumente weist (vgl. auch Heinze/Schmid/ Strünck 1997 mit ähnlichen Befunden). Offensichtlich wird freiwilliges Kontraktmanagement zur Stabilisierung etablierter und noch häufig zur weiteren Stärkung korporatistischer Arrangements eingesetzt. 
Abbildung 4: Vermehrte Übertragung von Aufgaben auf Dritte nach Einführung von Kontraktmanagement

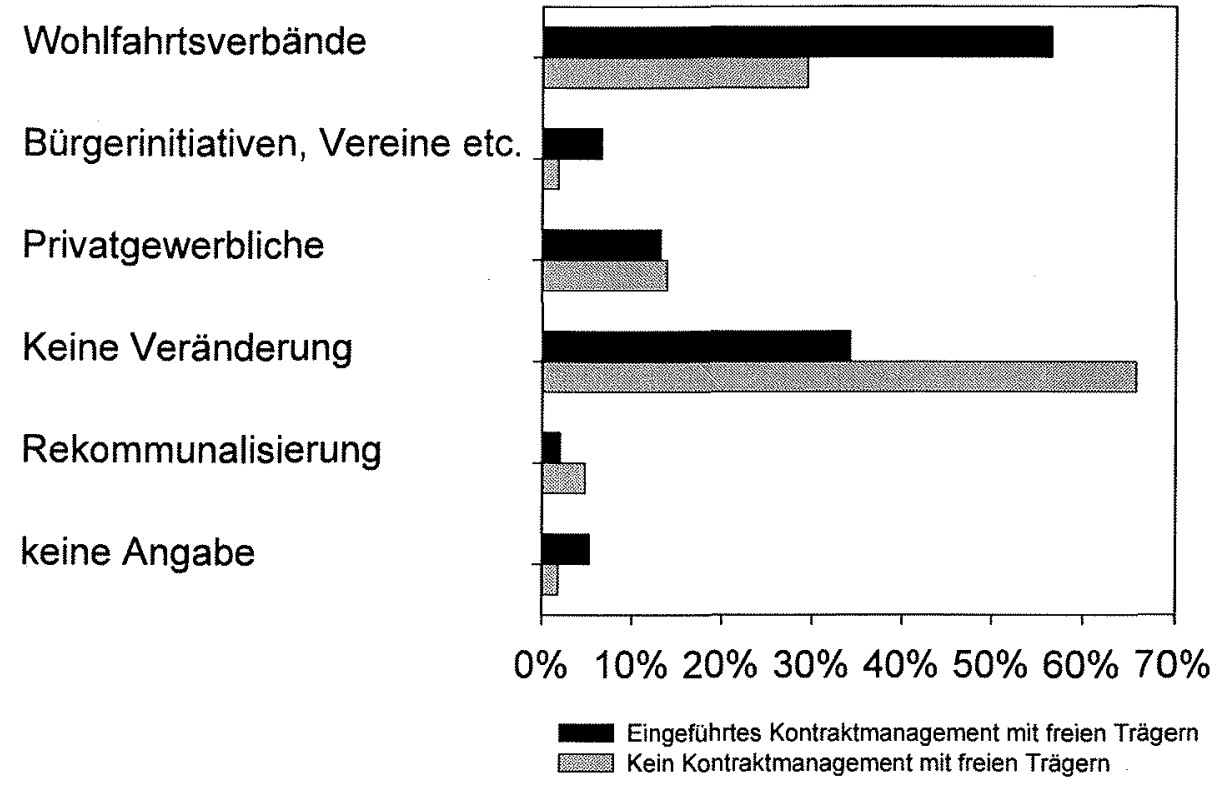

Quelle: HBS-Projekt „10 Jahre NSM“", Jugendamtsbefragung; $n=241$.

Quelle: HBS-Projekt „10 Jahre NSM“, Jugendamtsbefragung; $n=164$, nur Kommunen mit Leistungsverträgen gingen in die Auswertung ein.

Betrachtet man den zweiten potentiellen Ökonomisierungs- und Pluralisierungsimpuls, die Einführung von Leistungsvereinbarungen, zeigt sich ebenfalls zunächst eine erklärungsbedürftige Tendenz. Während das NSM in Westdeutschland weitaus häufiger umgesetzt wird als in Ostdeutschland, lässt sich beim neuen Instrument der Leistungsvereinbarungen die umgekehrte Tendenz feststellen. Gerade im Osten zeigt sich ein häufigerer Einsatz dieser - ebenfalls kontraktuellen - Steuerungsform. In über 50\% der ostdeutschen Jugendämter werden in der Mehrzahl der Fälle Zuwendungen durch Leistungsvereinbarungen und -verträge ersetzt. In weiteren rd. $25 \%$ der Jugendämtern immerhin in einigen Fällen. Die entsprechenden Anteile der westdeutschen Jugendämter betragen $40 \% \mathrm{bzw}$. $25 \%$ (NSM-Umfrage).

Dies könnte in verschiedener Hinsicht gedeutet werden: Entweder unterliegen die ostdeutschen Kommunen einem höheren Ökonomisierungsdruck, das Instrument der Leistungsvereinbarungen passt eher auf die ostdeutschen Gegebenheiten einer etwas pluraleren Trägerlandschaft oder die westdeutschen Verbände sind eher in der Lage, das neue Instrument abzuwehren. Fasst man Leistungsverträge und -vereinbarungen als Ökonomisierungsinstrument auf, wie dies in der Literatur häufig geschieht (vgl. Messmer 2007), müssten diese jedoch auch tatsächlich im Sinne einer größeren Markt- und Wettbewerbsorientierung eingesetzt werden. Das heißt, bei deren Abschluss müssten Vergleiche zwischen Anbietern unter Kosten- und Qualitätsaspekten durchgeführt werden. Bei den Antworten der Befragten zeigte sich allerdings, dass in den westdeutschen Kommunen, die Leistungsvereinbarungen eingeführt haben, in weniger als der Hälfte der Fälle solche Qualitäts- und Kostenvergleiche "teilweise“ angestellt werden (fasst man keine Antwort als Nichtdurchführung auf, was in diesem Fall nahe liegt). In der „Mehrzahl“ der Verträ- 
ge passiert dies nur in rd. $25 \%$. In den neuen Bundesländern liegen die entsprechenden Anteile mit knapp unter $60 \%$ bzw. $40 \%$ etwas höher. In großen Teilen scheinen also die Leistungsvereinbarungen nur eine Scheinökonomisierung darzustellen und die Beweggründe für deren Abschluss liegen wiederum in anderen Bereichen (die Vereinbarungen sind rechtlich nicht zwingend notwendig).

Letzter Schritt dieser Analyse stellt den Zusammenhang zwischen Leistungsverträgen und einer potentiellen Pluralisierung (vgl. Abb. 5) dar. Auch hier zeigt sich, dass in der Breite der Kommunen der Einsatz von Leistungsvereinbarungen eher zu einer Stärkung der verbandlichen Wohlfahrtspflege beigetragen hat. Andererseits ist hier auch ein deutlich höherer Anteil an Zusammenarbeit mit privatgewerblichen Trägern zu verzeichnen. Dies sind übrigens auch die Fälle, in denen tatsächlich Marktvergleiche angestellt werden und keine bloß formale Implementation der Leistungsverträge vorliegt.

Abbildung 5: Aufgabenübertragung nach Implementation von Leistungsverträgen

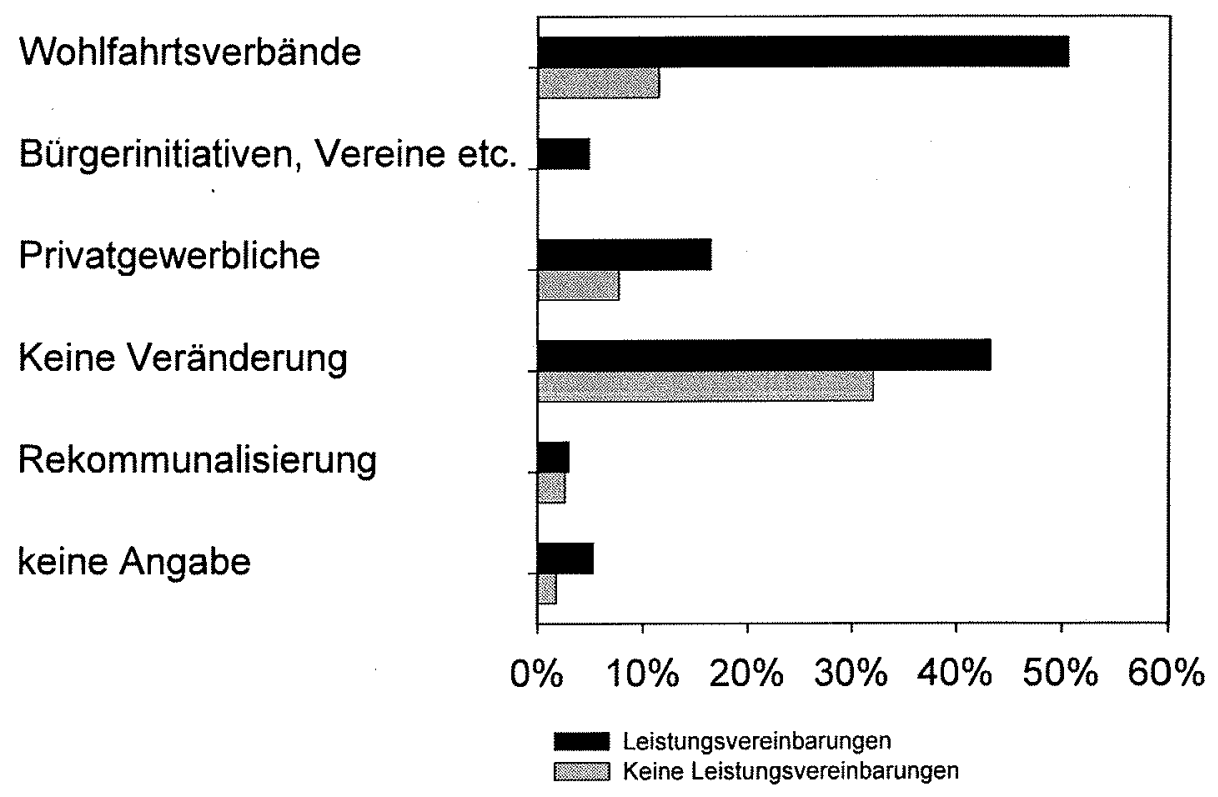

Quelle: HBS-Projekt „10 Jahre NSM“, Jugendamtsbefragung; $n=164$, nur Kommunen mit Leistungsverträgen gingen in die Auswertung ein.

Die Bilanz des Wandels der Trägerstrukturen und der Implementation neuer Regelungsinstrumente lässt sich also folgendermaßen zusammenfassen: Zwar wurden in einer Vielzahl der Kommunen neue Steuerungsformen (Kontraktmanagement) und auf eine Ökonomisierung der Wohlfahrtsarrangements zielende Vertragsinstrumente (Leistungsvereinbarungen) implementiert. Ein Wandel der Trägerstrukturen und eine tatsächliche Ökonomisierung der Vertragsverhältnisse lassen sich aber in den seltensten Fällen feststellen. Es wurden also auf breiter Front neue Spielregeln etabliert, ohne die Spieler auszuwechseln und auch ihr Spielgebaren zu verändern. Hierauf deuten auch mehrere in neueren Untersuchungen berichtete Befunde (vgl. Zimmer 2006 zur Einführung von Leistungsvereinbarungen in Münster und Jena; Merchel 2006 zur Implementation von Qualitätsentwicklungsvereinbarungen in NRW; Messmer 2007; Münder/Tammen 2003 zu Leistungsvereinbarungen) hin. 
Die präsentierten Analysen zeigen auf den ersten Blick widersprüchliche Ergebnisse, die jedoch bei eingehender Betrachtung einem deutlichen Muster folgen. Dabei zeigt sich ein Phänomen, das als ,halbierte Kontraktualisierung" gekennzeichnet werden kann. Dabei handelt es sich um eine - verglichen mit anderen Modernisierungselementen - häufige Implementation kontraktförmiger Vereinbarungen zwischen öffentlichen und freien Trägern im Feld der Jugendhilfe. Diese zeitigt jedoch nicht die ursprünglich in den Konzepten angelegten Folgen einer an Qualitäts- und Kostenaspekten orientierten Steuerung der lokalen Wohlfahrtsarrangements, sondern schlägt im Gegenteil häufig in das Gegenteil einer weiteren Konzentration der Leistungserstellung bei großen Trägern der etablierten Wohlfahrtsverbände um, für deren qualitative Steuerung keine adäquaten Instrumente implementiert werden.

\section{Gegenimplementation von unten: Wie lokale Akteure das "Angebot" neuer Instrumente strategisch nutzen}

Die Ergebnisse der quantitativen Betrachtungen scheinen zunächst die Beharrungsthese zu bestätigen. Gleichzeitig lässt sie jedoch entscheidende Fragen offen: Warum werden trotz offenkundigem Verfehlen der in den Instrumenten angelegten Ziele die neuen Instrumente rege eingeführt? Diese quantitativen Befunde führten zur Anschlussfrage, was für konkrete Implementationsweisen sich hinter diesem zunächst kontraintuitiven Zwischenergebnis verbergen. Dazu werden im Weiteren in Fallstudien Charakteristika der Implementationsweisen und wesentliche Faktoren zur Erklärung der unterschiedlichen eingeschlagenen Pfade herausgearbeitet. ${ }^{5}$

Die vier Fallstudien zeigen vier recht unterschiedliche Formen des Umgangs mit neuen Steuerungsformen und Kontraktmanagement auf. W1 verfolgt eine qualitätsorientierte Professionspolitik, die weitgehend dem vom BMSFJ unterstützten Leitbild eines ,,fachlich orientierten Qualitätswettbewerbs " entspricht. Hier liegen gewisse Pluralisierungstendenzen auf Seiten der Träger vor und die Leistungsbeziehungen werden konsequent auf Leistungsvereinbarungen umgestellt, in denen Leistungs-, Kosten- und Qualitätsziele vereinbart werden. Ausschlaggebend sind aber nicht Kosten- sondern Qualitätsfragen. Im Zentrum steht ein qualitäts- oder wirkungsorientiertes Controlling. Eine relativ gleichgewichtige und gleichberechtigte Rolle von fachlicher Ebene und zentraler Verwaltungsführung führten hier zu für beide Seiten tragfähigen Kompromissen, die zwar von ihrem Implementationsgrad her teilweise noch in den Kinderschuhen stecken, aber ein Potential für eine weitergehende Entwicklung auf dem eingeschlagenen Weg erkennen lassen.

W2 verfolgt demgegenüber eine rein legitimatorische Anpassung an von außen herangetragene Steuerungsansprüche. Unter legitimatorischer Anpassung werden Fälle gefasst, in denen zwar - meist auf Druck der Verwaltungsspitze - die Formalstruktur von Leistungsvereinbarungen und Kontrakten implementiert, diese aber nicht mit einer Kontraktkultur gefüllt werden, d.h. es wird weitgehend auf definierte Leistungs- und Qualitätsziele verzichtet. Durch hohe Führungsspannen und dadurch entstehende Informationsasymmetrien hat in W2 die Verwaltungsspitze keine objektiven Möglichkeiten, das Kontraktwesen auf der Fachebene zu durchschauen und kontrollierend einzugreifen. Hier findet eine inkrementale Anpassung an Anforderungen der Verwaltungsspitze statt, die aber keine Steuerungs- oder Konsolidierungsabsichten zur Folge hat. 
O1 stellt einen Fall recht weitgehender Ökonomisierung dar, wie er nur in sehr seltenen Fällen anzutreffen ist. Hier findet eine deutliche Pluralisierung auf Seiten der Träger statt und die Leistungsbeziehungen beruhen konsequent auf Leistungsvereinbarungen, in denen Leistungs-, Kosten- und Qualitätsfragen vereinbart werden. In der ausschreibungsbasierten Vergabe der Leistungen geben Kosten- und Qualitätskriterien den Ausschlag. Voraussetzung dafür war eine durchsetzungsfähige Verwaltungsführung, die unter starkem Haushaltsdruck arbeitet, eine schwache fachliche Ebene und eine nicht nach neokorporatistischen Mustern aufgestellte Trägerlandschaft vor Ort.

O2 stellt das Gegenbeispiel einer schwachen, durch die politische Konstellation (Kohabitation) kaum handlungsfähigen Verwaltungsführung dar, die sich gegen eine stark fachlich integrierte Fachverwaltung kaum durchsetzen kann. Diese nutzt die entstehenden Spielräume, um an den Interessen der Fachverwaltung an leistungsfähigen Trägern und den Bestandsinteressen der freien Träger orientierten Konzepten zu implementieren. Dies stellt einen exemplarischen Fall der Gegenimplementation dar.

An dieser Stelle kann keine ausführliche Fallrekonstruktion stattfinden (vgl. hierzu Grohs 2010, S. 197-234). Zur Frage, welche Implementationsweise sich durchsetzt, lassen sich aus den Fallstudien drei wesentliche Schlüsselvariablen ableiten:

Erste Schlüsselvariable ist der Druck der „,zentralen Steuerungspolitiker" (Banner 1984) auf die sozialpolitischen „Fachbruderschaften“ (ebd.), durch die Reform der Leistungsfinanzierung zur Haushaltskonsolidierung beizutragen. Dieser Druck hängt zentral von der Haushaltslage ab.

Zweite Schlüsselvariable ist die effektive Durchsetzung dieser Steuerungsambition der Verwaltungsspitze. Diese ist durch mehrere intervenierende Faktoren beeinflusst: Verfestigte neokorporatistische Strukturen, wie sie insbesondere in den alten Bundesländern vorzufinden sind, behindern die effektive Durchsetzung. Eine Schlüsselposition stellt offensichtlich die Besetzung des Sozialdezernenten als Bindeglied zwischen Steuerungsund Fachpolitikern dar: Agiert dieser unabhängig von den Verbandsinteressen und kann dennoch fachliche Vorgänge in den ihm untergeordneten Einheiten überblicken, steigt die Durchsetzungsfähigkeit der Verwaltungsspitze (Beispiele finden sich hier vor allem in Fallkommune O1; vgl. auch ein nicht benannter Kreis in Dahme u.a. 2005). In zahlreichen Kommunen ist die Sozialdezernentenposition jedoch mit Vertretern aus dem Verbändeumfeld besetzt (quantitative Angaben liegen meines Wissens nicht vor), die in der Lage sind diese externen Interessen zu mobilisieren (Insbesondere in O2). Zweitens sinkt die Durchsetzungsfähigkeit mit der Dezentralisierung von Verantwortungsstrukturen, wie es im NSM Programm war (vgl. Holtkamp 2007 am Beispiel kommunaler Haushaltspolitik). Diese verstärken tendenziell Informationsasymmetrien. Das NSM kann so (nur scheinbar paradoxerweise) zum Pluralisierungs- bzw. Ökonomisierungshindernis werden. Schließlich sind auch parteipolitische Konstellationen zu nennen, die ein Durchgreifen zentraler Steuerungspolitiker auf die Fachebene beeinflussen. In beiden Fällen ,vorsichtiger Pluralisierung" (W1 und O1) haben wir es in den Fallstudien mit klaren politischen Mehrheiten und z.T. einer Gleichläufigkeit der Parteizugehörigkeit des (Ober-) Bürgermeisters zu tun. In beiden Kommunen wurde nur vorsichtig dezentralisiert, jedoch mit betriebswirtschaftlichen Steuerungsinstrumenten eher zentralistischen Zuschnitts gearbeitet. Die entsprechenden Dezernenten gehören keinen fachlichen Netzwerken an und können eher als Statthalter der Verwaltungsspitze verstanden werden.

In den Fallkommunen mit subversiven Reaktionen (Gegenimplentation bzw. rein legitimatorische Anpassung) finden wir Kohabitationskonstellationen vor oder eine Auf- 
bauorganisation, die eine wirksame Kontrolle der Fachebene erschwert. Im Falle der größten „fachlichen Freiräume" finden wir sehr konfliktreiche Konstellationen im Rat, wo sich PDS/Linke und CDU gegenüberstehen. In solchen Konstellationen wird offensichtlich die Kontrolle der Fachverwaltung durch Politik und Verwaltungsspitze deutlich erschwert. Im anderen Fall ist die Führungsspanne auf Grund einer radikalen Reduzierung der Dezernate zu groß um wirksam in die Fachebene durchzugreifen. In beiden Fällen finden wir eine recht weitgehende Dezentralisierung der Ressourcenverantwortung, die jedoch in beiden Fällen nur unzureichend durch ein entsprechendes Controlling und Anreizmechanismen rückgekoppelt wird.

Dritte Schlüsselvariable ist die Existenz fachpolitischer Modernisierungskoalitionen, die fachliche Diskussionen im Umfeld sozialer Arbeit aufgreifen und offensiv in die Modernisierungsdebatte auf lokaler Ebene einbringen. Solche Koalitionen aus Fachakteuren innerhalb und außerhalb von Verwaltung und Trägern versuchen, die Herausforderungen von neuer Steuerung und Ökonomisierungsdruck fachpolitisch zu wenden (vgl. ausführlicher Grohs 2007, S. 249-255). Prominentestes Beispiel im Kontext dieses Beitrags ist die schon angeführte Sozialraumbudgetierung. Die Existenz einer solchen Modernisierungskoalition wird mit der Einbindung in neokorporatistische Netzwerke wahrscheinlicher, da viele Fachdiskurse in Arenen stattfinden, die durch die Spitzenverbände vorstrukturiert werden (Arbeitsgemeinschaft für Jugendhilfe; Deutscher Verein für öffentliche und private Fürsorge etc.). Wiederum kann die professionelle Herkunft des Sozialdezernenten förderlich sein und schließlich bietet ein allgemeiner Modernisierungsdiskurs (z.B. NSM) auch Gelegenheitsfenster fachpolitischer Modernisierung (vgl. Bogumil u.a. 2007, S. 8082). Solche Modernisierungskoalitionen können ihr Potential am weitesten entfalten, wenn sie sich möglichst dem Zugriff von Verwaltungsspitze und Kommunalpolitik entziehen können: „Die effektive Organisation eines Bündnisses zwischen Vertreter/innen einer fachlichen Sichtweise ist am ehesten möglich über eine Organisation, die bezogen auf diese Fragestellungen nicht in die klassische kommunalpolitische Gemengelage unterschiedlichster Strömungen eingebunden ist" (Hinte u.a. 1999, S. 141). Diese Bedingungen waren unter den Fallstädten insbesondere in $\mathrm{O} 2$ - befördert durch die unübersichtliche Gemengelage und starke fachpolitische Spieler auf Seiten des öffentlichen Trägers - zu finden. Mit Abstrichen gilt dies auch für W2.

Das stark vereinfachende Pfadmodell in Abbildung 6 fasst dieses Erklärungsmodell zusammen. Es geht davon aus, dass eine Pluralisierungs- und Ökonomisierungsstrategie nur durch Druck der Verwaltungsspitze und einen durchsetzungsfähigen Sozialdezernenten gegen die konterkarierenden Interessen der Fachverwaltung und die Verbandsvertreter erfolgen kann. Eine bloße legitimatorische Anwendung der Instrumente erfolgt in der Regel, wenn von außen Druck auf die Fachverwaltungen ausgeübt wird, von Seiten der Verwaltungsspitze aber keine Kompetenz vorliegt, das fachpolitisch vorstrukturierte Feld der Kinder- und Jugendhilfe zu durchblicken. Häufig geht es in der Kontraktgestaltung um die fachliche Definition von Bedarfslagen und die fachlich adäquate Definition von Kennziffern u.ä., die den Professionellen in den Fachämtern erhebliche Wissensvorsprünge gewähren. Eine dritte mögliche Reaktionsweise auf externen Druck ohne tatsächliche Durchsetzungsmacht ist die genannte Gegenimplementation der Instrumente. Eine solche Strategie hängt wesentlich von der ,Kreativität" und Kompetenz der Fachverwaltung ab. Diese steigt mit der Einbindung in fachpolitische Modernisierungskoalitionen. Eine solche Gegenimplementationsstrategie ist auch unabhängig vom Druck der Verwaltungsspitze denkbar; gerade unter Planbarkeitsgesichtspunkten ist sie in Ost- 
deutschland häufiger vorzufinden und wird hier gerade unter fachpolitischen Gesichtspunkten verfolgt. Der Residualfall der Beibehaltung des Status quo, so meine These (die ich in meinen Fallstudien nicht belegen kann, da der Fall nicht miteinbezogen ist), findet sich in Fällen wo wenig externer Druck existiert und auch unter fachpolitischen Gesichtspunkten kein Änderungsbedarf erkannt wird.

Abbildung 6: Pfadmodell zum Wandel lokaler Wohlfahrtsarrangements

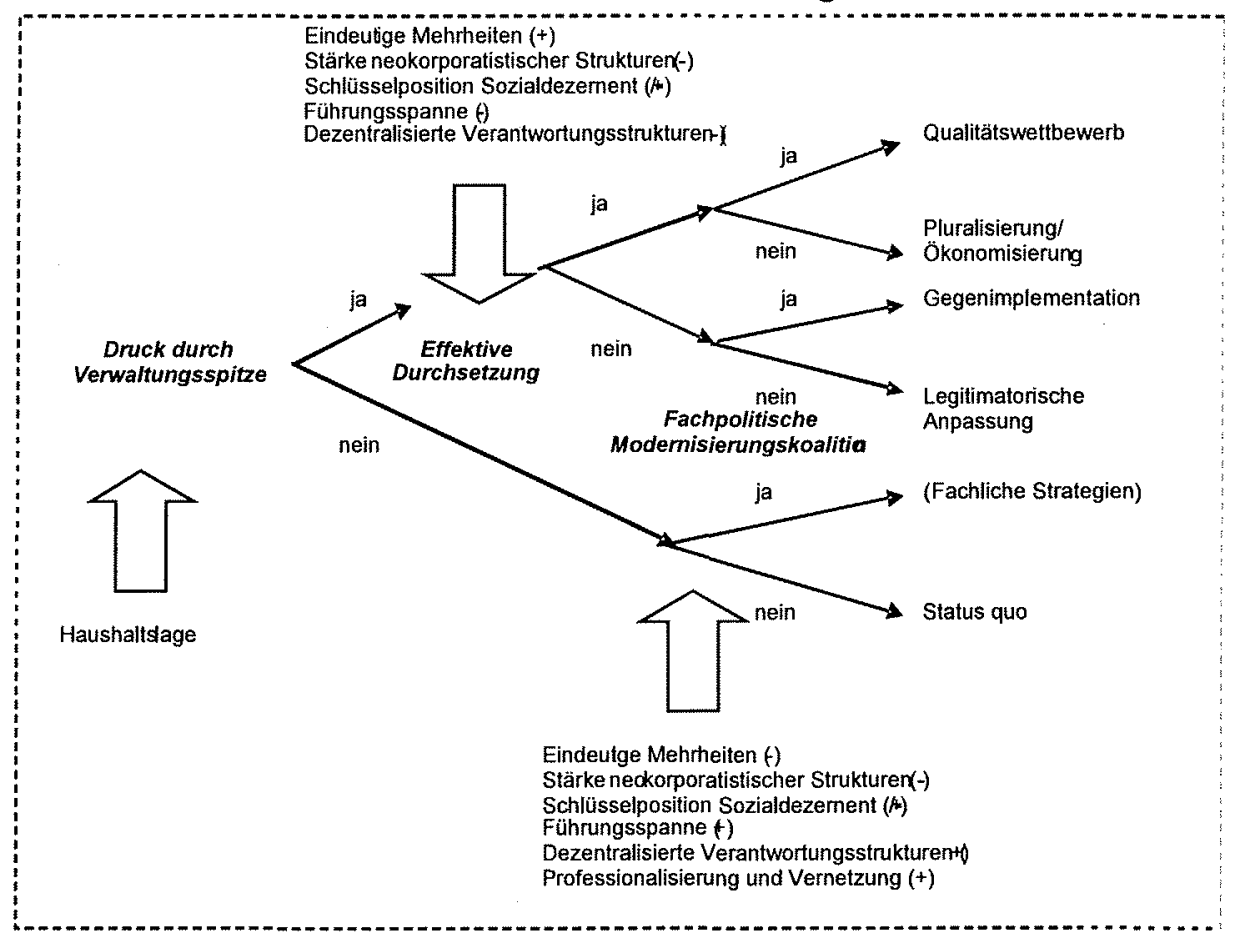

\section{Fazit}

Während in anderen Feldern sozialer Dienstleistungsproduktion Pluralisierungstendenzen vorzufinden sind sowie eine zunehmende Vermarktlichung festzustellen ist ${ }^{6}$ und auch in anderen bisher eher neokorporatistisch strukturierten Politikfeldern von "Postkorporatismus" bzw. neuem Pluralismus die Rede ist (vgl. Trampusch 2006; von Winter 2004; Heinze 2009), scheinen auf kommunaler Ebene geronnene Strukturen zwischen Verwaltung und Verbändelandschaft auf den ersten Blick eine erstaunliche Persistenz aufzuweisen. Dies deckt sich auch mit Erkenntnissen über die Einführung von Kontraktmanagement im Ausland.

Zieht man für eine zusammenfassende Interpretation auch die Ergebnisse anderer Forschungen heran ergibt sich das folgende Bild für die Mehrheit der deutschen Kommunen: Der Wandel in den Trägerstrukturen bleibt trotz Aufgabenverschiebungen marginal und standardisierte Qualitätssicherung bleibt weitgehend aus bzw. wird weiterhin durch pauschalisiertes Vertrauen und den Gerüchtemarkt vor Ort ersetzt (vgl. Abschnitt 5). Diese altvordere Art der Qualitätssicherung bleibt gerade aus Sicht der Kommunalpolitiker häufig abstrakten Kennzahlensystemen überlegen. Die lokalen Akteure wenden wie gesehen sogar eine erhebliche Kreativität auf, um eine der Stabilisierung der geronnenen Verhältnisse durch Aneignung des durch Pluralisierungsintentionen geschaffenen Werkzeug- 
kastens oder rein legitimatorische Anpassungen an Erwartungshaltungen anderer Steuerungsebenen $\mathrm{zu}$ erreichen. Wirkliche Wandlungstendenzen scheinen sich nur durch externen Druck und eher hierarchische Implementationsstrukturen durchsetzen zu lassen. Die etablierten Arrangements scheinen sich aus der Sicht der Akteure vor Ort bewährt zu haben. Häufig stehen Alternativen zu etablierten Akteuren auch schlicht nicht zur Verfügung. Die Positions- und Zugangsregeln bleiben weitgehend in Kraft: Weiterhin findet sich eine starke Verflechtung der Verbände mit der Sozialadministration und den örtlichen Parteien. Als eine wesentliche Schlüsselposition erwies sich der Sozialdezernent.

Die weit verbreitete „subversive Aneignung“ der Kontraktualisierung deutet jedoch darauf hin, dass die lokalen Fachbruderschaften durchaus Problembewusstsein und eigenständige Präferenzbildung zeigen. Die Akteure verhalten sich durchaus kalkulierend und nicht nach einer reinen Angemessenheitslogik. Einerseits können zur Begründung Bestandsinteressen angeführt werden. Es stellt sich heraus, dass Planungssicherheit gerade für kleinere Anbieter einen entscheidenden Faktor darstellt. Dieses Kalkül wird insbesondere in den für alle Seiten noch immer unsicheren ostdeutschen Wohlfahrtsarrangements relevant, wo durch den demographischen Wandel gerade erst etablierte Leistungsstrukturen neu in Frage gestellt werden. Leistungsvereinbarungen und Kontrakte werden von den Akteuren bewusst gewählt, da sie einen rechtlichen Rahmen bieten, in dem die Akteure ihre geteilten Ziele besser verwirklichen können als im alten System der Zuwendungsfinanzierung. Hier ist insbesondere die Möglichkeit mehrjähriger Leistungsvereinbarungen zu nennen, die den Trägereinrichtungen größere Planungssicherheit einräumt, da die in der Zuwendungsfinanzierung notwendige jährliche Genehmigung des Haushalts durch den Rat entfällt und gerade in unsicherer Haushaltslage Planungssicherheit schafft. Andererseits scheinen sich in vielen Fällen die schlichten Eigeninteressen der Verbände durchzusetzen und sich durch Kontraktmanagement Elemente einer weiteren Schließung des Wohlfahrtsmarktes herauszuschälen.

$\mathrm{Zu}$ zeigen, welche Auswirkungen die vorgestellten Reaktionsweisen auf die tatsächliche Leistungserbringung vor Ort haben, musste in diesem Beitrag außen vor bleiben und würde durch komplexe Wirkungsketten auch ein schwieriges Unterfangen. $\mathrm{Zu}$ vermuten ist, dass der wahrscheinlich positivste Effekt der Pluralisierungsstrategien in einer größeren Wahlfreiheit der Klienten zu sehen wäre (sofern diese bereit und in der Lage wären, von dieser Gebrauch zu machen). Potentiellen Effizienzgewinnen durch Wettbewerb stehen höhere Transaktionskosten gegenüber, die durch entfallende Eigenbeteiligung z.B. der kirchlichen Träger ergänzt werden. Unter fachlichen Gesichtspunkten ist zu diskutieren, ob eine Pluralisierung und damit auch Spezialisierung der Leistungen mit dem gegenläufigen Trend zu integrierten Hilfen und Casemanagement vereinbar ist. Aus einer politischen Steuerungsperspektive schließlich ist die Frage, ob die Steuerung über etablierte Vertrauensbeziehungen in vielen Kommunen nicht der effektivere Weg ist als über das selten funktionierende Kontraktmanagement. Andererseits könnten Transparenzgewinne, die durch effektiv durchgesetzte Qualitätsvereinbarungen erzielt werden, auch bei nicht stattfindender Pluralisierung einen Gewinn darstellen und der oft hinter den neokorporatistischen Beziehungen vermuteten Mauschelei vorbeugen.

\section{Anmerkungen}

1 Ich greife in diesem Abschnitt auf die Pfadabhängigkeitsdiskussion (vgl. North 1990, S. 92-96; Mahoney 2000; Pierson 2000) zurück und teile Beyers (2005) Appell an die Notwendigkeit einer Rückführung des ,Pfadabhängigkeitsarguments" auf Kausalmechanismen. Eine ausführliche Diskussion muss an anderer Stelle erfolgen. 
2 Unmittelbare Auslöserin war die kommunale Finanzmisere, die 1996 zu einer zweijährigen Deckelung der Jugendhilfeleistungen im Bereich der $\mathrm{HzE}$ führte und entsprechende Forderungen der kommunalen Spitzenverbände auslöste. Mittelbar war die Vorbildfunktion der Pflegeversicherung und anhaltende Kritik an den Wohlfahrtsverbänden (z.B. Seibel 1992) und dem „Wohlfahrtskartell“ (z.B. Monopolkommission 1998) mitverantwortlich.

3 Hauptquelle der Analyse zu Trägerstrukturen ist die Einrichtungsstatistik der Kinder- und Jugendhilfestatistik. Bei der Statistik über „Einrichtungen und tätige Personen in der Kinder- und Jugendhilfe“ handelt sich um eine Vollerhebung, die im Abstand von vier Jahren durchgeführt wird (vgl. Rauschenbach/Schilling 1997, S. 139-162). Es herrscht Auskunftspflicht für die Leitung der Einrichtungen. Zum Abgleich mit anderen Feldern der Wohlfahrtsproduktion werden zusätzlich die Daten der Pflegestatistik und andere Quellen herangezogen, die jeweils gesondert ausgewiesen werden.

4 Das Forschungsprojekt „10 Jahre Neues Steuerungsmodell - Evaluation kommunaler Verwaltungsmodernisierung" wurde von der Hans-Böckler-Stiftung gefördert und von der Kommunalen Gemeinschaftsstelle (KGSt) unterstützt (Laufzeit 2004-2006). Zu den Ergebnissen vgl. Bogumil u.a. 2007. Den folgenden Betrachtungen liegen Teilergebnisse einer bundesweiten schriftlichen Befragung von Jugendamtsleitungen zu Grunde, die von Ende Januar bis Juni 2005 durchgeführt wurde. Dabei handelte es sich um eine Vollerhebung aller Jugendämter in deutschen Städten und etwa $2 / 3$ der deutschen Landkreise. Der Rücklauf zum 30.06 .05 belief sich auf 242 Fragebögen, was einer Rücklaufquote von $46,8 \%$ entspricht.

5 Die Kommunen werden im Weiteren anonymisiert. Ausgewählt wurden daher zwei westdeutschen Kommunen mit einerseits pluralisierter Trägerstruktur (W1), andererseits stabiler Trägerstruktur (W2) sowie zwei ostdeutschen Pendants (O1 plural, O2 stabil). Detaillierte Fallbeschreibungen finden sich in Grohs 2010. Paradebeispiel ist die ambulante Pflege, vgl. Schneiders 2010.

\section{Literatur}

Angerhausen, Susanne/Backhaus-Maul, Holger/Offe, Claus/Olk, Thomas/Schiebel, Martina, 1998: Überholen ohne einzuholen. Freie Wohlfahrtspflege in Ostdeutschland, Opladen: Westdeutscher Verlag.

Backhaus-Maul, Holger/Olk, Thomas, 1994: Von Subsidiarität zu "Outcontracting": Zum Wandel der Beziehungen von Staat und Wohlfahrtsverbänden in der Sozialpolitik, in: Streeck, Wolfgang (Hrsg.), Staat und Verbände. PVS Sonderheft 25, Opladen: Westdeutscher Verlag, S. 100-135.

Banner, Gerhard, 1984: Kommunale Steuerung zwischen Gemeindeordnung und Parteipolitik, in: Die Öffentliche Verwaltung 37 (9), S. 364-372.

Beinecke, Richard/Goodman, Maury/Lockhart, Amy, 1997: The Impact of Managed Care on Massachusetts Mental Health and Substance Abuse Providers, in: Administration in Social Work, 21, S. 41-53.

Beyer, Jürgen, 2005: Pfadabhängigkeit ist nicht gleich Pfadabhängigkeit. Wider den impliziten Konservatismus eines gängigen Konzepts, in: Zeitschrift für Soziologie 34 (1), S. 5-21.

Bönker, Frank/Wollmann, Helmut, 2006: Public Sector Reforms and Local Governments in Germany: The Case of Local Social Policy, in: Hoffmann-Martinot, Vincent/Wollmann, Hellmut (Hrsg.): State and Local Government Reforms in France and Germany. Divergence and Convergence, Wiesbaden: VS, S. 189-206.

Boeßenecker, Karl-Heinz/Trube, Achim/Wohlfahrt, Norbert (Hrsg.), 2000: Privatisierung im Sozialsektor. Rahmenbedingungen, Verlaufsformen und Probleme der Ausgliederung sozialer Dienste, Münster: Votum.

Boeßenecker, Karl-Heinz/Trube, Achim/Wohlfahrt, Norbert (Hrsg.), 2001: Verwaltungsreform von unten? Lokaler Sozialstaat im Umbruch aus verschiedenen Perspektiven, Münster: Votum.

Bogumil, Jörg/Holtkamp, Lars, 2006: Kommunalpolitik. Eine policyorientierte Einführung, Wiesbaden: VS. Bogumil, Jörg/Grohs, Stephan/Kuhlmann, Sabine/Ohm, Anna K., 2007: Zehn Jahre Neues Steuerungsmodell - eine Bilanz kommunaler Verwaltungsmodernisierung, Berlin: Edition Sigma.

Bundesministerium für Familie, Senioren, Frauen und Jugend (BMFSFJ), 2002: Elfter Kinder- und Jugendbericht. Bericht über Lebenssituation junger Menschen und die Leistungen der Kinder- und Jugendhilfe in Deutschland, Berlin: BMFSFJ.

Bußmann, Ulrike/Esch, Karin/Stöbe-Blossey, Sybille, 2003: Neue Steuerungsmodelle - Frischer Wind im Jugendhilfeausschuss? Die Weiterentwicklung der neuen Steuerungsmodelle: Tendenzen und Potenziale am Beispiel der Jugendhilfe, Opladen: Leske und Budrich. 
Dahme, Heinz-Jürgen/Kühnlein, Gertrud/Wohlfahrt, Norbert/Burmester, Monika, 2005: Zwischen Wettbewerb und Subsidiarität. Wohlfahrtsverbände unterwegs in die Sozialwirtschaft, Berlin: Edition Sigma.

Evers, Adalbert/Olk, Thomas (Hrsg.), 1996: Wohlfahrtspluralismus. Vom Wohlfahrtsstaat zur Wohlfahrtsgesellschaft, Opladen: Westdeutscher Verlag.

Ever's, Adalbert/Rauch, Ulrich/Stitz, Uta, 2002: Von öffentlichen Einrichtungen zu sozialen Unternehmen. Hybride Organisationsformen im Bereich sozialer Dienstleistungen, Berlin: Edition Sigma.

Grohs, Stephan, 2007: Reform der Jugendhilfe zwischen Neuer Steuerung und Professionalisierung. Eine Bilanz nach 15 Jahren Modernisierungsdiskurs, in: Zeitschrift für Sozialreform 53 (3), S. 247-274.

Grohs, Stephan, 2010: Modernisierung kommunaler Sozialpolitik. Anpassungsstrategien im Wohlfahrtskorporatismus, Wiesbaden: VS.

Heinze, Rolf G., 1981: Verbändepolitik und „Neokorporatismus“. Zur politischen Soziologie organisierter Interessen, Opladen: Westdeutscher Verlag.

Heinze, Rolf G., 2009: Rückkehr des Staates? Politische Handlungsmöglichkeiten in unsicheren Zeiten, Wiesbaden: VS.

Heinze, Rolf G./Olk, Thomas, 1981: Die Wohlfahrtsverbände im System sozialer Dienstleistungsproduktion. Zur Entstehung und Struktur der bundesrepublikanischen Verbändewohlfahrt, in: Kölner Zeitschrift für Soziologie und Sozialpsychologie 33(1), S. 94-114.

Heinze, Rolf G./Schmid, Josef/Strünck, Christoph, 1997: Zur politischen Ökonomie der sozialen Dienstleistungsproduktion. Der Wandel der Wohlfahrtsverbände und die Konjunkturen der Theoriebildung, in: Kölner Zeitschrift für Soziologie und Sozialpsychologie, S. 242-271.

Heinze, Rolf G./Strünck, Christoph, 2000: Social Service Delivery by Private and Voluntary Organisations in Germany, in: Wollmann, Hellmut/Schröter, Eckhard (Hrsg.), Comparing Public Sector Reforms in Britain and Germany, Aldershot: Alsgate, S. 284-303.

Hinte, Wolfgang/Litges, Gerd/Springer, Werner, 1999: Soziale Dienste: Vom Fall zum Feld. Soziale Räume statt Verwaltungsbezirke, Berlin: Edition Sigma.

Johnson, Norman/Jenkinson, Sandra/Kendall, Ian/Bradshaw, Yvonne/Blackmore, Martin, 1998: Regulating for Quality in the Voluntary Sector, in: Journal of Social Policy, 27, S. 307-328.

Kaufmann, Franz-Xaver, 2003: Varianten des Wohlfahrtsstaats. Der deutsche Sozialstaat im internationalen Vergleich, Frankfurt/Main: Suhrkamp.

Kulbach, Roderich/Wohlfahrt, Norbert, 1996: Modernisierung der öffentlichen Verwaltung? Konsequenzen für die freie Wohlfahrtspflege, Freiburg: Lambertus.

Mahoney, James, 2000: Path Dependence in Historical Sociology, in: Theory and Society 29, S. 507-548.

Mamier, Jasmin/Seckinger, Mike/Pluto, Liane/van Santen, Eric/Zink, Gabriela, 2002: Organisatorische Einbettung von Jugendhilfeaufgaben in der Kommunalverwaltung, in: Sachverständigenkommission Elfter Kinder- und Jugendbericht (Hrsg.), Materialien zum elften Kinder- und Jugendbericht, Band 1, München: DJI, S. 265-318.

March, James G./Olsen, Johan P., 1989: Rediscovering Institutions. The Organizational Basis of Politics, New York/London: Free Press.

Merchel, Joachim, 2006: § 78b SGB VIII als Instrument zur Qualitätsentwicklung in der Erziehungshilfe? Ergebnisse einer Inhaltsanalyse von Qualitätsentwicklungsvereinbarungen, in: Zeitschrift für Kindschaftsrecht und Jugendhilfe 2/2006, S. 78-89.

Messmer, Heinz, 2007: Jugendhilfe zwischen Qualität und Kosteneffizienz, Wiesbaden: VS.

Meyer, Dirk, 2008: Die Kinder- und Jugendhilfe im Spannungsverhältnis zwischen Korporatismus und Markt, in: Sozialer Fortschritt, S. 208-217.

Meyer, John W./Rowan, Brian, 1991: Institutionalized Organizations: Formal Structure as Myth and Ceremony, in: DiMaggio, Paul J./Powell, Walter W. (Hrsg.), The New Institutionalism in Organizational Analysis, Chicago: Chicago University Press, S. 41-62.

Möhring-Hesse, Matthias, 2008: Verbetriebswirtschaftlichung und Verstaatlichung. Die Entwicklung der Sozialen Dienste und der Freien Wohlfahrtspflege, in: Zeitschrift für Sozialreform, 54, S. 141160.

Monopolkommission, 1998: Marktöffnung umfassend verwirklichen. Zwölftes Hauptgutachten, BadenBaden: Nomos.

Münder, Johannes, 1998: Von der Subsidiarität über den Korporatismus zum Markt?, in: Neue Praxis, S. 3-12. 
Münder, Johannes/Tammen Britta, 2003: Die Vereinbarungen nach $\S$ 78a ff. SGB VIII. Bestandsaufnahme und Analyse der Leistungs-, Entgelt- und Qualitätsentwicklungsvereinbarungen sowie der Rahmenverträge (Eine Studie im Auftrag des Bundesministeriums für Familien, Senioren, Frauen und Jugend.), Berlin: Verein für Kommunalwissenschaften, S. 11-81.

North, Douglas C., 1990: Institutions, Institutional Change and Economic Performance, Cambridge: CUP.

Ostrom, Elinor, 1986: An Agenda for the Study of Institutions, in: Public Choice 48, S. 3-25.

Ostrom, Elinor, 1999: Institutional Rational Choice. An Assessment of the Institutional Analysis and Development Framework, in: Sabatier, Paul (Hrsg.), Theories of the Policy Process, Boulder: Westview Press, S. 35-71.

Pabst, Stefan, 1998: Interessenvermittlung im Wandel. Wohlfahrtsverbände und Staat im Postkorporatismus, in: Arbeitskreis Non-Profit-Organisationen (Hrsg.), Nonprofit-Organisationen im Wandel Ende der Besonderheiten oder Besonderheiten ohne Ende? Frankfurt /M: Eigenverlag, S. 177-197.

Pierson, Paul, 2000: Increasing Returns, Path Dependency and the Study of Politics, in: American Political Science Review 94, S. 251-267.

Pluto, Liane, 2005: Verwaltungsmodernisierung bei Jugendämtern - Impulse, Entwicklungen, Bewertungen, in: Archiv für Wissenschaft und Praxis der sozialen Arbeit 36 (2), S 20-36.

Pluto, Liane/Gragert, Nicola/van Santen, Eric/Seckinger, Mike, 2007: Kinder- und Jugendhilfe im Wandel. Eine empirische Strukturanalyse, München: DJI.

Rauschenbach, Thomas/Schilling, Matthias, 1997: Die Kinder- und Jugendhilfe und ihre Statistik. Band I: Einführung und Grundlagen, Neuwied: Luchterhand.

Romzek, Barbara/Johnston, Jocelyn, 2002: Effective Contract Implementation and Management: A Preliminary Model, in: Journal of Public Administration Research and Theory 12, S. 423-453.

Ruflin, Regula Franziska, 2006: Wohlfahrtsstaatliches Kontraktmanagement. Die Verhandlung und Umsetzung von Leistungsverträgen als Herausforderung für Nonprofit-Organisationen, Bern: Haupt.

van Santen, Eric, 1998: „Output" und „outcome“" der Implementierung Neuer Steuerung, in: Neue Praxis 28 (1), S. 36-49.

Schneiders, Katrin, 2010: Vom Altenheim zum Seniorenservice. Institutioneller Wandel und Akteurkonstellationen im sozialen Dienstleistungssektor, Baden-Baden: Nomos.

Seckinger, Mike/Weigel, Nicole/van Santen, Eric/Markert, Andreas, 1998: Situation und Perspektiven der Jugendhilfe - eine empirische Zwischenbilanz, München: DJI.

Seibel, Wolfgang, 1992: Funktionaler Dilettantismus. Erfolgreich scheiternde Organisationen im 'Dritten Sektor' zwischen Markt und Staat, Baden-Baden: Nomos.

Smith, Steven Rathgeb/Smyth, Judith, 1996: Contracting for Services in a Decentralized System, in: Journal of Public Administration Research and Theory, 6, 277-296.

Trampusch, Christine, 2006: Postkorporatismus in der Sozialpolitik - Folgen für Gewerkschaften, in: WSI-Mitteilungen 59 (6), S. 347-352.

Van Slyke, David M., 2003: The Mythology of Privatization in Contracting for Social Services, in: Public Administration Review, 63, 296-315.

von Winter, Thomas, 2004: Vom Korporatismus zum Lobbyismus. Zum Paradigmenwechsel in der Interessenvermittlungsforschung, in: Zeitschrift für Parlamentsfragen, H. 4/2004, S. 761- 776.

Wollmann, Hellmut, 1983: Implementation durch Gegenimplementation von unten, in: Mayntz, Renate (Hg.): Implementation politischer Programme II, Opladen: Westdeutscher Verlag, S. 168-196.

Zimmer, Annette, 2006: Vereine - Zivilgesellschaft Konkret. 2. Auflage. Wiesbaden: VS.

Anschrift des Autors:

Dr. Stephan Grohs, Universität Konstanz, Fachbereich für Politik und Verwaltungswissenschaft, Lehrstuhl für vergleichende Policy-Forschung und Verwaltungswissenschaft, Universitätsstraße 10,78464 Konstanz

E-Mail: stephan.grohs@uni-konstanz.de 